The border collision normal form with stochastic switching surface

Glendinning, Paul

2012

MIMS EPrint: 2012.76

Manchester Institute for Mathematical Sciences

School of Mathematics

The University of Manchester

\footnotetext{
Reports available from: http://eprints.maths.manchester.ac.uk/

And by contacting: The MIMS Secretary

School of Mathematics

The University of Manchester

Manchester, M13 9PL, UK
} 


\title{
The border collision normal form with stochastic switching surface
}

\author{
Paul Glendinning \\ Centre for Interdisciplinary Computational and Dynamical Analysis (CICADA) and \\ School of Mathematics, University of Manchester, Manchester, M13 9PL, U.K. \\ Email: p.a.glendinning@manchester.ac.uk
}

\begin{abstract}
The deterministic border collision normal form describes the bifurcations of a discrete time dynamical system as a fixed point moves across the switching surface with changing parameter. If the position of the switching surface varies randomly, but within some bounded region, we give conditions which imply that the attractor close to the bifurcation point is the attractor of an Iterated Function System. The proof uses an equivalent metric to the Euclidean metric because the functions involved are never contractions in the Euclidean metric. If the conditions do not hold then a range of possibilities may be realized, including local instability, and some examples are investigated numerically.
\end{abstract}

PACS: 05.40.-a, 05.45.-a

Keywords: non-smooth bifurcation, attractor, border collision, stochastic dynamics, switched system

\section{Introduction}

Many problems involve systems in which discrete and continuous components interact. In switched systems the dynamical systems describing the evolution changes whenever some threshold (the switching surface) is crossed. Switched systems are common in control and digital applications, but examples are also found in more standard electronic circuits [1], biological modelling [3] and mechanical problems [7]. There is a growing body of results describing the bifurcations of deterministic models [4]. In some situations, more realistic modelling would need to take account of different sources of 
uncertainty: the dynamical systems describing the evolution of variables may have a stochastic element, and the switching surface itself may be subject to fluctuations. Initial results from a dynamical systems perspective on the former case have been obtained recently by Simpson et al [13, 14]. Whilst there are studies of more general stochastic hybrid systems in the computer science literature (e.g. $[5,6]$ ), this tends to restrict attention to describing properties of the class of solutions obtained rather than giving a detailed description of dynamics.

In this paper we consider the effect of random fluctuations in the switching surface, whilst retaining a deterministic description of the dynamics in the different regions defined by the switching surface. Such fluctuations may have mechanical origins (e.g. panel flutter or poorly fitted boards), or be caused by measurement techniques (e.g. if the signal is sampled digitally and the transition occurs if the measurement is within some tolerance of the theoretical switching surface), or arise as a result of intrinsic variation (e.g. thermal effects).

To keep the analysis as simple as possible we restrict attention to a modification of the discrete time model derived by Nusse and Yorke [12] to describe the bifurcations that occur when a fixed point of a map intersects the switching surface at some value of the parameter. This model, the border collision normal form, has been used to help understand a variety of phenomena in switched systems [1], and the fact that it has a discrete time variable means that complications inherent in the description and definition of a continuously varying switching surface in continuous time systems, such as multiple intersections with the trajectory in a short space of time, are avoided. In keeping with the mechanical and measurement sources of noise described above we assume that the switching surface varies randomly but within specified bounds. Without the boundedness assumption (see equation (6) of section 2) the system is of a more standard type, where the probability of applying either one of the maps defining the deterministic border collision normal form is always non-zero. Thus if the assumption of bounded fluctuations is not imposed, the system is a standard Iterated Function System if the maps are contractions with respect to some metric, and a more general random map otherwise. One of the main aims of this paper is to investigate how the assumption of bounded fluctuations allows for both deterministic features and random features as the bifurcation parameter is varied. The advantage of the simple model presented here is that concrete results are possible, and in section 4 we show that the stochastically switched border collision system 

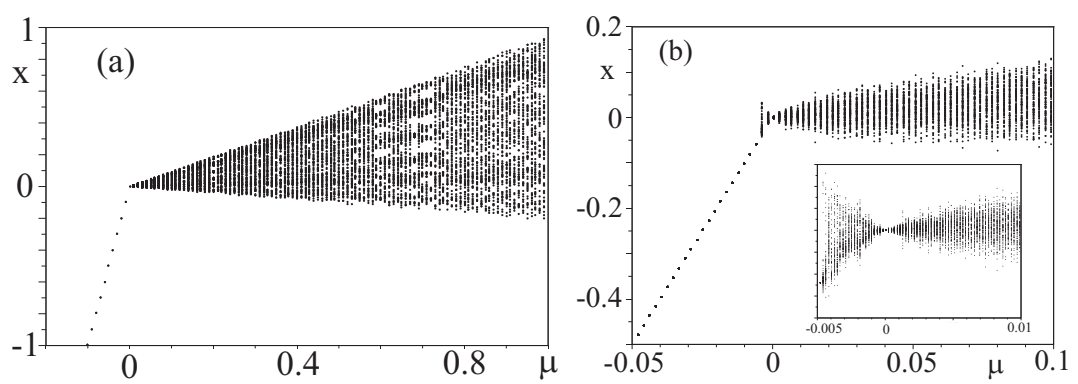

Figure 1: Parameters $T_{R}=-0.3 ; D_{R}=1.1 ; T_{L}=0.1 ; D_{L}=-0.8$ (as defined in section 2). (a) Bifurcation diagram (100 values of the $x$ variable of a trajectory for different values of $\mu$ ) for the deterministic border collision normal form showing an abrupt transition from a stable fixed point to a chaotic state. (b) Bifurcation diagram for the border collision normal form with stochastic switching surface taking values between -0.05 and 0.05 (100 values for each chosen value of $\mu$ after 1000 iterations to get convergence). The inset shows detail of the structure near $\mu=0$; note the appearance of the complex attractor before the bifurcation value of the deterministic normal form. The variation of the switching surface, $\epsilon$ of $(6)$, is 0.05 .

of section 2 can have regions of parameters where the dynamics is described by an Iterated Function System.

Figure 1 shows the bifurcation diagram of an example that does not satisfy the tidy contraction conditions of section 4. In Figure 1a a deterministic border collision bifurcation creates a chaotic attractor, whose size goes to zero at the bifurcation from a stable fixed point. In the stochastically switched version of Figure 1b there is a clear intermediate region before the emergence of the chaotic border collision attractor (see the inset of Figure 1b), and we will look at the structure of this bifurcation and some other examples in section 5. One of the features of these examples is that it is easy to produce unbounded motion close to the bifurcation point. This seems to be a stochastic analogue of the 'dangerous bifurcations' of [10], where, even in the deterministic case it is possible to find unbounded orbits at the bifurcation value itself, though there are local attractors at neighbouring parameter values.

The stochastic model is defined in the next section. In section 3 we give the background on contractions and Iterated Function Systems needed to prove the main result. In particular, a choice of an equivalent metric to the Euclidean metric is described. In this metric the maps defining the border collision normal form are contractions for some parameter values (they are 
never contractions in the Euclidean metric). The main result, showing the appearance of IFS attractors, is proved in section 4 and in section 5 some comments are made together with numerical simulations to indicate how the behaviour is changed if the conditions of the main result do not hold.

\section{The stochastically switched model}

The deterministic border collision normal form is a continuous, piecewise affine map and we will consider here only the two-dimensional case, although very similar remarks hold in higher dimensions. Let

$$
\mathcal{R}_{0}=\left\{(x, y)^{T} \mid x \leq 0\right\}, \quad \mathcal{R}_{1}=\left\{(x, y)^{T} \mid x \geq 0\right\}
$$

and if $\left(x_{n}, y_{n}\right)^{T} \in \mathcal{R}_{k}$ define a discrete time evolution by

$$
\left(\begin{array}{l}
x_{n+1} \\
y_{n+1}
\end{array}\right)=\left(\begin{array}{cc}
T_{k} & 1 \\
-D_{k} & 0
\end{array}\right)\left(\begin{array}{l}
x_{n} \\
y_{n}
\end{array}\right)+\left(\begin{array}{c}
\mu \\
0
\end{array}\right)
$$

where $T_{k}$ and $D_{k}$ are constants, $k=0,1$, and $\mu$ is a real parameter. This is a deterministic dynamical system with switching surface $x=0$. It can be derived as the linear approximation for the dynamics of a switched system which is continuous across the switching surface and which has a fixed point on the switching surface if $\mu=0$ [12]. Much is known about the attractors of such maps both because of their application in nonsmooth bifurcation theory [12? ], but also because they are very natural simple maps of the plane to investigate [11]. Note that in this formulation the dynamics depends only on the sign of $\mu$ if the other parameters are fixed, since by a simple scaling $\mu$ can be restricted to $\{-1,0,1\}$. This scale invariance will disappear in the stochastic model below.

It will be convenient to rewrite (1), (2) in terms of two affine maps $F_{k}$ : $\mathbb{R}^{2} \times \mathbb{R} \rightarrow \mathbb{R}^{2}$ where for $z \in \mathbb{R}^{2}$

$$
F_{k}(z ; \mu)=A_{k} z+m, \quad k=0,1
$$

with

$$
m=(\mu, 0)^{t} \quad \text { and } \quad A_{k}=\left(\begin{array}{cc}
T_{k} & 1 \\
-D_{k} & 0
\end{array}\right)
$$

$k=0,1$, where the superscript $t$ denotes the transpose of a matrix or vector (lower case to avoid confusion with the coefficients $T_{k}$ of the matrix $A_{k}$ ). In 
terms of $F_{0}$ and $F_{1}$ the border collision normal form is

$$
z_{n+1}= \begin{cases}F_{0}\left(z_{n} ; \mu\right) & \text { if } x_{n} \in \mathcal{R}_{0} \\ F_{1}\left(z_{n} ; \mu\right) & \text { if } x_{n} \in \mathcal{R}_{1}\end{cases}
$$

A simple stochastic model which includes the effects of fluctuations in the switching surface is obtained by replacing the switching surface $x=0$ by a random set of (independent) surfaces. To simplify matters further we assume that these surfaces lie in a bounded strip near $x=0$ and that they are graphs over $y$, so

$$
x=g_{n}(y),
$$

and there exists $\epsilon>0$ such that for all $n$ and $y$

$$
\left|g_{n}(y)\right|<\epsilon \text {. }
$$

Now define, as in (1),

$$
\mathcal{R}_{n, 0}=\left\{(x, y)^{T} \mid x<g_{n}(y)\right\}, \quad \mathcal{R}_{n, 1}=\left\{(x, y)^{T} \mid x \geq g_{n}(y)\right\},
$$

and consider the dynamical system generated by (2) with $k=0$ if $\left(x_{n}, y_{n}\right)^{T} \in$ $R_{n, 0}$ and $k=1$ if $\left(x_{n}, y_{n}\right)^{T} \in R_{n, 1}$, i.e.

$$
z_{n+1}= \begin{cases}F_{0}\left(z_{n} ; \mu\right) & \text { if } x_{n}<g_{n}\left(y_{n}\right) \\ F_{1}\left(z_{n} ; \mu\right) & \text { if } x_{n} \geq g_{n}\left(y_{n}\right)\end{cases}
$$

This is the system whose dynamics is described below. (Note that we have made an arbitrary decision to include the switching surface in $\mathcal{R}_{n, 1}$, though many other conventions could have been used. The central results below are not changed by the choices made.) By scaling, the threshold $\epsilon$ can be chosen to take any fixed positive value, but to emphasize that we are envisaging small fluctuations here, the value $\epsilon=0.05$ is used in all the numerical simulations reported here.

The independence of these graphs implies that given any sequence

$$
y_{0}, y_{1}, y_{2}, \ldots
$$

then the values

$$
g_{0}\left(y_{0}\right), g_{1}\left(y_{1}\right), g_{2}\left(y_{2}\right), \ldots
$$

are also independent. This means that to compute a single (random) trajectory it is enough to generate an independent set of real random variables 
$\left(\xi_{n}\right)$ with $\left|\xi_{n}\right|<\epsilon$ and apply the border collision normal form with $k=0$ if $x_{n}<\xi_{n}$ and $k=1$ if $x_{n} \geq \xi_{n}$. Of course, if realizations of different initial conditions from the same random run are sought for, the situation becomes considerably more complicated and a more precise definition of the class of random functions being considered is necessary. Since we will not be looking at this aspect of the problem, all realizations of trajectories can be computed using a sequence of real, independent random variable, which we will also take to be identically distributed.

One further point is worth noting. Unlike the original border collision normal form, the maps (for a given $g_{n}$ ) are not continuous across the switching surface. This could be addressed by a stochastic modification of the functions $F_{0}$ and $F_{1}$ (a shift in the $x$-variable), but we will not follow this line of argument here.

\section{Contractions, Iterated Function Systems and Equivalent Met- rics}

In this section we recall some basic facts about Iterated Function Systems and contractions. If $\mathcal{R} \subseteq \mathbb{R}^{2}$, then a function $f: \mathbb{R}^{2} \rightarrow \mathbb{R}^{2}$ is a contraction on $\mathcal{R}$ in the metric $d(.,$.$) if there exists a constant c \in(0,1)$ such that for all $z, z^{\prime} \in \mathcal{R}$

$$
d\left(f(z), f\left(z^{\prime}\right)\right) \leq c d\left(z, z^{\prime}\right)
$$

An IFS (or Iterated Function System) [2] on a compact subset $\mathcal{R} \subseteq \mathbb{R}^{2}$ with metric $d(.,$.$) is a finite set of continuous contractions \left\{f_{1}, \ldots, f_{m}\right\}$ on $\mathcal{R}$. Define a dynamics on subsets, $S$, of $\mathcal{R}$ by

$$
F(S)=\cup_{r=1}^{m} f_{r}(S)
$$

and $F^{p}(S)=F\left(F^{p-1}(S)\right), p \geq 2$. One of the central results of the theory of IFSs is that there exists a unique $F$-invariant set $\Lambda \subset \mathbb{R}^{2}$ such that $F(\Lambda)=\Lambda$, and

$$
\Lambda=\cup_{r=1}^{m} f_{r}(\Lambda) .
$$

Iteration of a single point $x_{0} \in \mathbb{R}^{2}$ under randomly chosen sequences of $f_{r}$ tend to $\Lambda$, so $\Lambda$ can be seen as the attractor of the random dynamical system obtained by iterating a point with the functions $f_{r}$ where at each iteration the next function to apply is chosen randomly.

An affine function $F(z)=A z+b$ for some constant real invertible matrix $A$ is contracting iff all the eigenvalues of the symmetric matrix $A^{T} A$ lie inside 
the unit circle (note that this is not the same as saying that the stationary point of the matrix is stable). If the eigenvalue of $A^{T} A$ with largest modulus has modulus $s^{2}<1$ say, $(s>0)$ then this implies that

$$
\|A u\|^{2}=u^{T} A^{T} A u \leq s^{2}\left(u^{T} u\right)=s^{2}\|u\|^{2}, \quad \text { for all } u \in \mathbb{R}^{2}
$$

where\|.\| denotes the standard Euclidean norm in $\mathbb{R}^{2}$, from which it is easy to deduce that if $v=A u+b$ and $v^{\prime}=A u^{\prime}+b$ for some constant vector $b$ then

$$
\left\|v-v^{\prime}\right\| \leq s\left\|u-u^{\prime}\right\|
$$

or that the vector function $A u+b$ is a contraction with contraction coefficient $s$. It may come as a little surprise that affine functions with a matrix of the form used in (2) is never a contraction in the standard Euclidean metric, and this means that it is necessary to work with alternative metrics. Since this adds an extra level of technical difficulty to this paper the point is worth making formally.

Lemma 1. If

$$
A=\left(\begin{array}{cc}
T & 1 \\
-D & 0
\end{array}\right)
$$

then $A$ is not a contraction in the Euclidean metric.

Proof: By direct calculation

$$
A^{T} A=\left(\begin{array}{cc}
T^{2}+D^{2} & T \\
T & 1
\end{array}\right)
$$

and since this is symmetric the eigenvalues are real. In this case an elementary calculation shows that if the eigenvalues are less than one then $\operatorname{Tr}\left(A^{T} A\right)<2$ and $1-\operatorname{Tr}\left(A^{T} A\right)+\operatorname{det}\left(A^{T} A\right)>0$. But the second of these equations implies that $T^{2}<0$, so $A$ cannot be a contraction.

A metric $d$ is equivalent to the Euclidean metric iff an open set in the topology defined by one metric is also open in the topology defined by the other. It is fairly straightforward to show that if $|$.$| denotes the standard$ real (one-dimensional) Euclidean metric and $\alpha>0$ then the function $d_{\alpha}$ : $\mathbb{R}^{2} \times \mathbb{R}^{2} \rightarrow \mathbb{R}$ defined by

$$
d_{\alpha}\left(\left(x_{1}, y_{1}\right),\left(x_{2}, y_{2}\right)\right)=\alpha\left|x_{2}-x_{1}\right|+\left|y_{2}-y_{1}\right|
$$


is a metric, and it is equivalent to the Euclidean metric in the plane. The advantage of this metric is that matrices of the form (10) can be contractions in this metric. An open ball about a point is (geometrically) a rhombus.

Lemma 2. If $A$ is a matrix of the form (10) and $|T|+|D|<1$ then there exists $\alpha>0$ such that $A$ is a contraction in the metric $d_{\alpha}$.

Proof: Given $z_{k}=\left(x_{k}, y_{k}\right), k=1,2, A z_{k}=\left(T x_{k}+y_{k},-D x_{k}\right)$ and so

$$
\begin{aligned}
d_{\alpha}\left(A z_{1}, A z_{2}\right) & =\alpha\left|T x_{2}-T x_{1}+y_{2}-y_{1}\right|+|D|\left|x_{2}-x_{1}\right| \\
& \leq(\alpha|T|+|D|)\left|x_{2}-x_{1}\right|+\alpha\left|y_{2}-y_{1}\right| \\
& \leq \alpha\left(\left(|T|+\alpha^{-1}|D|\right)\left|x_{2}-x_{1}\right|+\left|y_{2}-y_{1}\right|\right) .
\end{aligned}
$$

Thus

$$
d_{\alpha}\left(A z_{1}, A z_{2}\right) \leq \alpha\left(\alpha\left|x_{2}-x_{1}\right|+\left|y_{2}-y_{1}\right|\right)=\alpha d_{\alpha}\left(z_{1}, z_{2}\right)
$$

provided

$$
\alpha>|T|+\alpha^{-1}|D|, \quad \text { i.e. } \quad \alpha^{2}-|T| \alpha-|D|>0,
$$

and $A$ is a contraction for $d_{\alpha}$ if such a value can be chosen with $\alpha \in(0,1)$. The larger real root of $\alpha^{2}-|T| \alpha-|D|=0$ is strictly less than one provided $1-|T|-|D|>0$, so if this inequality is satisfied then such an $\alpha$ can be found and hence $A$ is a contraction in the metric $d_{\alpha}$.

Note that the condition $|T|+|D|<1$ specifies a square in the $(T, D)$ plane with corners $(-1,0),(0,1),(1,0)$ and $(0,-1)$. This square contains all those parameters for which the fixed point of the affine map is stable if $D<0$, but only a subset of those for which the fixed point is stable if $D>0$.

In the equations defined in (2) the dynamics is determined by the two maps $F_{0}$ and $F_{1}$ of (3). The comments above make it possible to show that $F_{0}$ and $F_{1}$ are contractions under some conditions and so the pair is an IFS. A bound for the size of the invariant set of (9) can also be found.

Lemma 3. Suppose $D_{k} \neq 0,\left|T_{k}\right|+\left|D_{k}\right|<1$ and let $\alpha_{k}$ be the larger root of the quadratic equation $s^{2}-\left|T_{k}\right| s-\left|D_{k}\right|=0, k=0,1$. Then the pair $\left\{F_{0}, F_{1}\right\}$ of (3) is an IFS in the metric $d_{c}$, for $c$ in the non-empty interval $\left(\max \left(\alpha_{0}, \alpha_{1}\right), 1\right)$. Moreover given any $u^{*} \in \mathbb{R}^{2}$, the attractor of the IFS lies in the region defined by

$$
d_{c}\left(u_{*}, p\right)<K\left(\frac{1+c}{1-c}\right)
$$


where $K$ is the maximum of the distances between $u^{*}$ and the fixed points of the two maps.

Proof: That the pair $\left\{F_{0}, F_{1}\right\}$ forms an IFS follows immediately from the definition of the affine functions $F_{k}$, Lemma 2) and the proof below that there is a compact absorbing region. This part of the proof can be found in $[8,9]$ for example, but since less detail is needed than given in these references the argument will be given from first principles.

Pick $c$ as in the statement of the lemma. Since $D_{k} \neq 0$, there exist unique fixed points of the two maps, $z_{k}^{*}, k=0,1$, which lie a 'distance' $d^{*}=d_{c}\left(z_{0}^{*}, z_{1}^{*}\right)$ apart. Suppose that the attractor of the IFS is $\Lambda$. Then $\Lambda=F_{0}(\Lambda) \cup F_{1}(\Lambda)$ (cf. (9)). So suppose that $\Lambda$ is contained within a ball $B$ (i.e. rhombus) of radius $R$ about the reference point $u^{*}$, and let

$$
\ell_{k}=d_{c}\left(u^{*}, z_{k}^{*}\right), \quad k=0,1 .
$$

Then all points in $B$ are within a distance $\ell_{k}+R$ of $z_{k}^{*}$. Hence for any $u \in B$,

$$
d_{c}\left(F_{k}(u), z_{k}^{*}\right)=d_{c}\left(F_{k}(u), F_{k}\left(z_{k}^{*}\right)\right) \leq c d_{c}\left(u, z_{k}\right) \leq c\left(\ell_{k}+R\right)
$$

and so

$$
d_{c}\left(F_{k}(u), u^{*}\right) \leq d_{c}\left(F_{k}(u), z_{k}^{*}\right)+d_{c}\left(z_{k}^{*}, u^{*}\right) \leq c\left(\ell_{k}+R\right)+\ell_{k} .
$$

The ball $B$ is invariant if $d_{c}\left(F_{k}(u), u^{*}\right) \leq R$ for all $u \in B$ and this is assured if the right hand side of (14) is less than $R$, i.e. if

$$
c\left(\ell_{k}+R\right)+\ell_{k} \leq R, \quad k=0,1 .
$$

Rearranging this shows that $B$ is an absorbing set (and hence contains the unique attractor $\Lambda$ ) provided

$$
R \geq \max \left(\ell_{0}, \ell_{1}\right)\left(\frac{1+c}{1-c}\right) .
$$

Obviously, the bound on $R$ defined above could be improved in all sorts of ways. By choosing $u^{*}=\frac{1}{2}\left(z_{0}^{*}+z_{1}^{*}\right)$ the distances $\ell_{0}$ and $\ell_{1}$ are equal and we may dispense with the maximum in (15). 


\section{Stochastic switching surface with contracting normal forms}

The deterministic maps (3) of the border collision normal form have fixed points $\left(x_{k}^{*}, y_{k}^{*}\right), k=0,1$, where

$$
x_{k}^{*}=\frac{\mu}{1-T_{k}+D_{k}}, \quad y_{k}^{*}=-D_{k} x_{k}^{*} .
$$

If $\left|T_{k}\right|+\left|D_{k}\right|<1, k=0,1$, (the conditions for the maps to be contractions for an appropriate equivalent metric as shown in the previous section, Lemma 2), then the denominator $1-T_{k}+D_{k}$ is positive and so $x_{0}^{*}$ is in $x<0$ if $\mu<0$ and $x_{1}^{*}$ is in $x>0$ if $\mu>0$. In other words, the two fixed points cannot both exist as fixed points of the border collision normal form (4) for any given parameter, and the transition described by the border colliion normal form is from a stable fixed point in $x<0$ if $\mu<0$, to a stable fixed point in $x>0$ if $\mu>0$, i.e. there is effectively no bifurcation. It is known [1] that there are parameters of the deterministic normal form such that this change (or no change!) occurs together with the creation of other stable sets, for example more complicated periodic orbits can coexist with at least one of the stable fixed points. However, for the choice of parameters made here, this added complication cannot happen. We are not aware of this result being stated in the literature, so the proof will be included here.

Lemma 4. If $\left|T_{k}\right|+\left|D_{k}\right|<1$ and $D_{k} \neq 0, k=0,1$, then the deterministic border collision normal form has a stable fixed point in $x<0$ if $\mu<0$ and a stable fixed point in $x>0$ if $\mu>0$. These are the unique attractors of the system.

Proof: As before, let $F_{k}, k=0,1$ denote the affine maps (3) of the border collision normal form. We will show that the deterministic border collision normal form is a continuous contraction (for an appropriate equivalent metric) in a compact region, from which the existence and uniqueness of the attracting fixed points follows using standard results. Let $\alpha_{k}$ be the larger root of $\alpha^{2}-\left|T_{k}\right| \alpha-\left|D_{k}\right|=0$, and note that $\alpha_{k} \in(0,1)$; let $s^{*}=\max \left(\alpha_{0}, \alpha_{1}\right)$.

The existence of an absorbing region for the deterministic border collision normal form is a corollary of the equivalent result (Lemma 3 ) for the corresponding IFS. Restricting to this absorbing region, if $z$ and $z^{\prime}$ are both in either $x<0$ or $x>0$ then $F_{0}$ and $F_{1}$ are both contracting with respect to the metric $d_{c}$ with $s^{*}<c<1$ by Lemma 2. Now suppose that $z=(x, y)$ 
with $x<0$ and $z^{\prime}=\left(x^{\prime}, y^{\prime}\right)$ with $x^{\prime}>0$ and let $v=\left(0, v^{*}\right)$ be the point on the switching surface $x=0$ on the straight line between $z$ and $z^{\prime}$. Then, dropping the argument $\mu$ in $F_{k}$ for the moment,

$$
\begin{aligned}
d_{c}\left(F_{0}(z), F_{1}\left(z^{\prime}\right)\right) & \leq d_{c}\left(F_{0}(z), F_{0}(v)\right)+d_{c}\left(F_{1}(z), F_{1}(v)\right) \\
& \leq c d_{c}(z, v)+c d_{c}\left(z^{\prime}, v\right)=c\left(d_{c}(z, v)+d_{c}\left(v, z^{\prime}\right)\right)=c d_{c}\left(z, z^{\prime}\right)
\end{aligned}
$$

where we have used the triangle inequality together with the continuity of the border collision normal form across $x=0$ ( so $F_{0}(v)=F_{1}(v)$ if the $x$ coordinate of $v$ is zero) and the fact that if points are collinear then the distance (measured by the metric $d_{c}$ ) between the endpoints equals the sum of the distance between those points and the intermediate point (an equality that is easily verified by direct calculation). Thus for this choice of parameters the deterministic border collision normal form is a continuous contraction and hence has a unique fixed point which attracts all iterates.

We now turn our attention to the noisy switched system of section 2 . The main result is that if $|\mu|$ is sufficiently small then the border collision normal form with noisy switching has an IFS attractor.

Theorem 5. Consider the border collision normal form with noisy switching (8) as described in section 2. Fix $\epsilon>0$ in (6) and assume that $\left|T_{k}\right|+\left|D_{k}\right|<1$ and $D_{k} \neq 0, k=0,1$. If $|\mu|$ is sufficiently large then there is a stable fixed point outside the region $[-\epsilon, \epsilon]$ of fluctuations. If $|\mu|$ is sufficiently small then the attractor is the attractor of the IFS defined by the two maps making up the border collision normal form.

Proof: The first statement is trivial: the fixed point of (16) lies in $x<-\epsilon$ if $\mu<-\left(1-T_{0}+D_{0}\right) \epsilon$ and in $x>0$ if $\mu>\left(1-T_{1}+D_{1}\right) \epsilon$.

Let $s^{*}=\max \left(\alpha_{0}, \alpha_{1}\right)$ be as in the proof of Lemma 4 and fix $c \in\left(s^{*}, 1\right)$. Choose $u^{*}=\frac{1}{2}\left(z_{0}^{*}+z_{1}^{*}\right)$ in the proof of Lemma 3 so that $u^{*}$ has $x$-coordinate $x^{*} \mu$ where

$$
x^{*}=\frac{1}{2}\left(\frac{1}{1-T_{0}+D_{0}}+\frac{1}{1-T_{1}+D_{1}}\right) \text {. }
$$

Thus

$$
\ell_{0}=\ell_{1}=d_{c}\left(z_{0}^{*}, u^{*}\right)=|\mu| Q
$$

where $Q$ is a positive constant (a function of the $T_{k}$ and $D_{k}$ but not $\mu$ ) which is easy enough to write down explicitly but whose precise form adds nothing 
to the argument. By Lemma 3 the absorbing region for the IFS generated by $F_{0}$ and $F_{1}$ is $p \in \mathbb{R}^{2}$ such that

$$
d_{c}\left(u^{*}, p\right) \leq|\mu| Q\left(\frac{1+c}{1-c}\right) .
$$

Our choice of $u^{*}$ makes it easy to calculate the range of $x$-coordinates of such points: they lie in the range

$$
x^{*} \mu-|\mu| \frac{Q}{c}\left(\frac{1+c}{1-c}\right) \leq x \leq x^{*} \mu+|\mu| \frac{Q}{c}\left(\frac{1+c}{1-c}\right)
$$

where $x^{*}$ is given by (17). Thus if

$$
|\mu|<\left(x^{*}+\frac{c}{Q}\left(\frac{1-c}{1+c}\right)\right)^{-1} \epsilon
$$

the entire absorbing region lies in the region in which the boundary fluctuates and so asymptotically there is, at each iteration, a positive probability of applying either $F_{0}$ or $F_{1}$ and so the attractor in the absorbing region is the attractor of the corresponding IFS.

Given more information about the random fluctuation of the switching surface more can be said about the distribution from which the maps $F_{0}$ and $F_{1}$ are chosen. Suppose, as argued in section 2, that given any sequence of $y$ values $\left(y_{n}\right)$ the random variables $\left(g_{n}\left(y_{n}\right)=\xi_{n}\right)$ are independent and identically distributed. Then a realization of the stochastically switched system takes the form, with $z_{n}=\left(x_{n}, y_{n}\right)$,

$$
z_{n+1}= \begin{cases}F_{0}\left(z_{n} ; \mu\right) & \text { if } x_{n}<\xi_{n} \\ F_{1}\left(z_{n} ; \mu\right) & \text { if } x_{n} \geq \xi_{n}\end{cases}
$$

If the $\xi_{n}$ are uniformly distributed on $[-\epsilon, \epsilon]$ and $|\mu|$ is small enough so that the IFS determines the attractor (i.e. $\left|x_{n}\right|<\epsilon$ for all sufficiently large $n$ ) then for sufficiently large $n$

$$
\operatorname{Prob}\left(x_{n}<\xi_{n}\right)=\frac{x_{n}+\epsilon}{2 \epsilon}
$$

and so $F_{0}$ is applied with probability $\frac{x_{n}+\epsilon}{2 \epsilon}$ and $F_{1}$ is applied with probability $\frac{x_{n}-\epsilon}{2 \epsilon}$. As $|\mu|$ tends to zero then $\left|x_{n}\right|$ lies in a smaller and smaller band around 


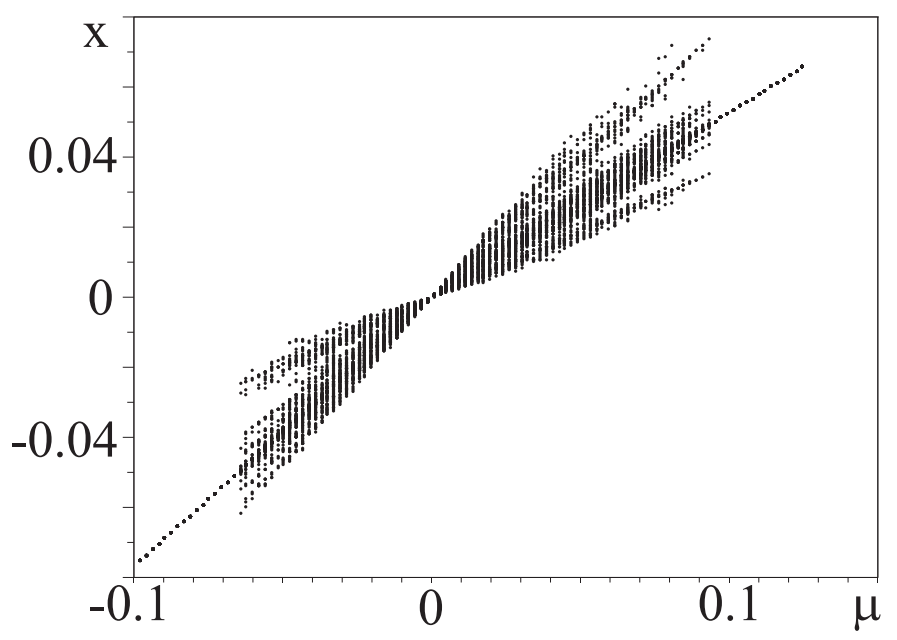

Figure 2: $T_{R}=-0.3 ; D_{R}=0.6 ; T_{L}=0.2 ; D_{L}=0.5$. Bifurcation diagram (100 values of the $x$ variable of a trajectory for different values of $\mu$ ) for the border collision normal form with stochastic switching surface taking values between -0.05 and 0.05 .

$x=0$ and so the IFS tends to a fair (equal probability) IFS. Note that if $\mu=0$ then $z_{n} \rightarrow 0$ for all initial $z_{0}$ (the composition of two contractions with the same fixed point). A similar analysis is possible for different choices of the distribution of the $\xi_{n}$.

Figure 2 shows a bifurcation diagram for the border collision normal form with noisy switching, showing the transition from a fixed point to a region dominated by the IFS and back again. Note that we have not been able to say much about the transition between the two (a question of how solutions are distributed on the attractor of the IFS when not all realizations of combinations of the maps $F_{0}$ and $F_{1}$ are possible). Figure 3 shows a couple of examples of the sort of attractors that can be observed in the IFS region.

\section{Other stochastic transitions}

Even if $\left\{F_{0}, F_{1}\right\}$ is not an IFS for a metric $d_{c}$ because at least one of them is not a contraction, random applications of the pair may still generate a bounded attractor. This attractor can also be the attractor of the border collision normal form with appropriate noisy switching. However, numerical simulations suggest that there are many cases for which no such bounded attractors exist and the local dynamics diverges for small $\| \mu \mid$ even if the 

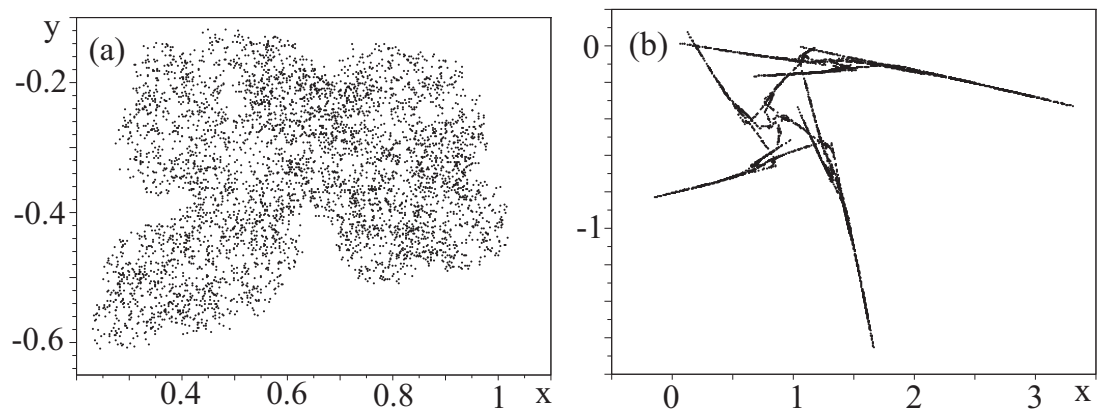

Figure 3: Attractors calculated using the equal probability IFS. (a) $T_{R}=-0.3 ; D_{R}=0.6$; $T_{L}=0.2 ; D_{L}=0.5$ (the same constants as in Figure 2) with 5000 iterates on the attractor shown. (b) As (a) but with $T_{R}=0.3 ; D_{R}=0.5 ; T_{L}=0.8 ; D_{L}=0.1$.

deterministic border collision normal form has attractors. Such divergence can also be seen in the deterministic case, where the behaviour at $\mu=0$ can be divergent even if there are attractors if $|\mu| \neq 0$. This phenomenon has been described as a dangerous bifurcation [10], so in the noisy case the instability appears to occur over a range of values of $\mu$ and not just at $\mu=0$. In applications this means that the solutions leave the small neighbourhood of the fixed point on which the border collision normal form is a good (linearized) model, and that nonlinear terms are needed in order to understand the asymptotic dynamics of solutions.

Figure 4 shows details of the attractor of the noisy border collision normal form with the same constants as that of Figure 1. For sufficiently small $|\mu|$ there is relatively little structure, but as $\mu$ increases the attractor develops a hole and becomes more like the annular attractor of the deterministic border collision normal form. For more negative $\mu$ the attractor is just a fixed point, so this is not shown. Some examples of other attractors that can be observed are shown in Figure 5.

\section{Conclusion}

To interpret the dynamics of deterministic hybrid models in applications, the extent to which dynamic features are robust to noise needs to be understood. In this paper we have examined one aspect of this problem, where the stochasticity is in the position of a switching surface, illustrating this with the border collision normal form in two dimensions. By using a suitably cho- 

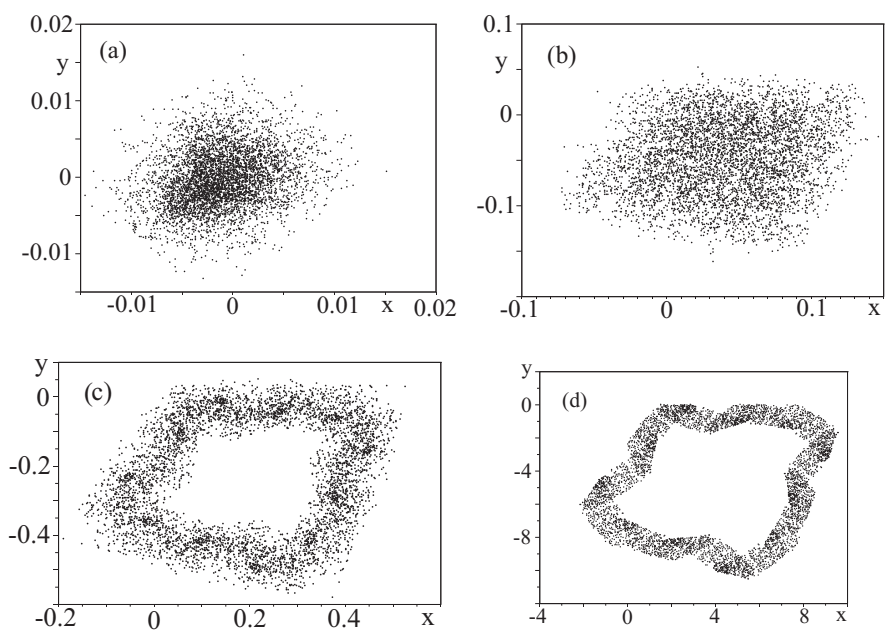

Figure 4: $T_{R}=-0.3 ; D_{R}=1.1 ; T_{L}=0.1 ; D_{L}=-0.8$, as in the example of Figure 1. 5000 iterates are shown after 1000 iterates to approach the attractor for each run at different values of $\mu$. (a) $\mu=-0.001$; (b) $\mu=0.1$; (c) $\mu=0.5$; and $\mu=10$ (stochastic switching surface restricted to $-0.05<x<0.05)$.
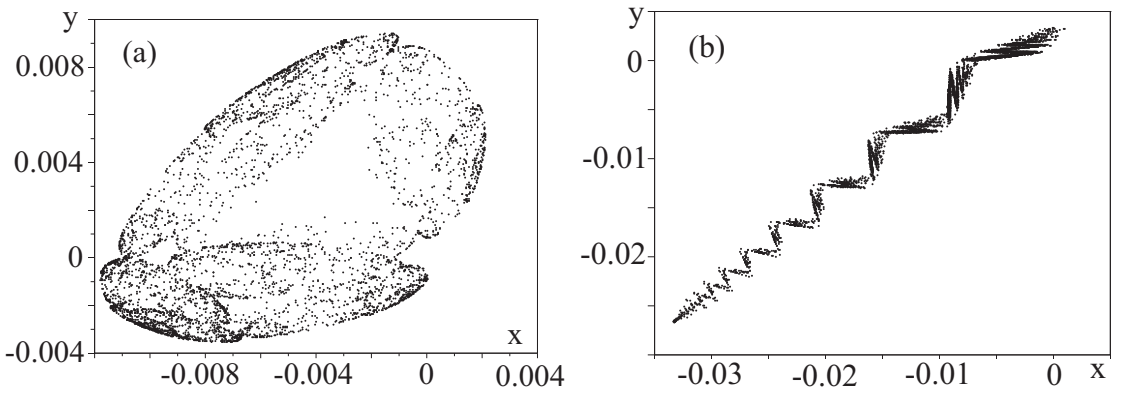

Figure 5: Attractors calculated using the stochastically switched border collision normal form showing different forms of the possible attractor, chosen for aesthetic just as much as scientific reasons. (5000 iterations after 10000 unplotted iterations to ensure that the attractors are shown; the stochastic switching surface is restricted to $-0.05<x<0.05$.) (a) $T_{R}=-0.8 ; D_{R}=0.8 ; T_{L}=-0.3 ; D_{L}=-0.3$ and $\mu=-0.01$. (b) $T_{R}=-1.1$; $D_{R}=0.1 ; T_{L}=-0.1 ; D_{L}=-0.8$ and $\mu=0.001$. 
sen equivalent metric we have shown that for a range of parameters, the noise generates an attractor which is equivalent to the attractor of an IFS when the bifurcation parameter is sufficiently close to its bifurcation value. Numerical investigations at parameters that do not satisfy the contraction condition suggest that whilst these conclusions continue to hold in some cases, it is also possible that motion can become unbounded close to the bifurcation point, and so the approximations leading to the border collision normal form break down.

Many variants of the model proposed here are possible; the assumption that the switching surface always lies in a given strip could be relaxed, and different distributions for the switching surface could be investigated. The combination of noisy switching and noisy dynamics would also be worth exploring. Moreover, it would be instructive to have a better understanding of the transition between the essentially deterministic behaviour at large $|\mu|$ and the IFS behaviour at small $|\mu|$.

Despite these caveats, the features described here are likely to remain important in systems where the dominant noise is in the switching mechanism and the deterministic dynamics is described by the border collision normal form.

Acknowledgements: I am grateful to Younes Chahlaoui for a useful conversation about equivalent metrics. This work was partially funded by EPSRC grant EP/E050441/1.

\section{References}

[1] S. Banerjee and C. Grebogi (1999) Border collision bifurcations in twodimensional piecewise smooth maps, Phys. Rev. E $594052-4061$.

[2] M.F. Barnsley and S. Demko (1985) Iterated function systems and the global construction of fractals, Proc. Roy. Soc. A 399 243-275.

[3] H. de Jong, J-L. Gouzé, C. Hernandez, M. Page, T. Sari and J. Geiselmann (2004) Qualitative simulation of genetic regulatory networks using piecewise-linear models, Bull. Math. Biol. 66 301-340.

[4] M. di Bernardo, C. Budd, A.R. Champneys, P. Kowalczyk (2008) Piecewise-smooth Dynamical Systems Theory and Applications, Applied Mathematical Sciences, vol. 163, Springer, London. 
[5] H.A.P. Blom and J. Lygeros (eds.) (2006) Stochastic Hybrid Systems, LNCIS, vol. 337, Springer, Berlin.

[6] L.M. Bujorianu (2012) Stochastic Reachability Analysis of Hybrid Systems, Communications and Control Engineering Series, Springer, London.

[7] H. Dankowicz and A.B. Nordmark (2000) On the origin and bifurcations of stick-slip oscillations, Physica D 136 280-302.

[8] S. Dubec and R. Hamzaoui (1994) On the diameter of the attractor of an IFS, Math. Rep. Acad. Sci. (Royal Society of Canada) 16 85-90.

[9] A. Edalat, D. Sharp and L. While (1997) Bounding the Attractor of an IFS, Information Processing Letters 64 197-202.

[10] M.A. Hassouneh, E.H. Ebed and H.E. Nusse (2004) Robust dangerous border-collision bifurcations in piecewise smooth systems, Phys. Rev. Lett. 92:070201.

[11] C. Mira, L. Gardini, A. Barugola and J.C. Cathala (1996) Chaotic Dynamics in Two-Dimensional Noninvertible Maps, World Scientific, Singapore.

[12] H.E. Nusse and J.A. Yorke (1992) Border-collision bifurcation including period two to period three for piecewise-smooth systems, Physica D 57 $39-57$.

[13] D.J.W. Simpson and R. Kuske (2012) Stochastically Perturbed Sliding Motion in Piecewise-Smooth Systems, arXiv: 1204.5792v1.

[14] D.J.W. Simpson, S.J. Hogan and R. Kuske (2012) Stochastic Regular Grazing Bifurcation, arXiv:1207.2703v2. 